\title{
Linaclotide Attenuates Visceral Organ Crosstalk: Role of Guanylate Cyclase-C Activation in Reversing Bladder-Colon Cross-Sensitization
}

\author{
Ehsan N. Mohammadi, Casey O. Ligon, Ada Silos-Santiago, Pei Ge, Caroline Kurtz, \\ Carolyn Higgins, Gerhard Hannig, and Beverley Greenwood-Van Meerveld
}

Oklahoma Center for Neuroscience (E.N.M., C.O.L., B.G.-V.M.), Department of Physiology (B.G.-V.M.), and Veterans Affairs Medical Center (B.G.-V.M.), University of Oklahoma Health Science Center, Oklahoma City, Oklahoma; and Ironwood Pharmaceuticals, Cambridge, Massachusetts (A.S.-S., P.G., C.K., C.H., G.H.)

Received February 16, 2018; accepted March 21, 2018

\begin{abstract}
Bladder pain syndrome (BPS) is poorly understood; however, there is a female predominance and comorbidity with irritable bowel syndrome (IBS). Here we test the hypothesis that linaclotide, a guanylate cyclase-C (GC-C) agonist approved for the treatment of IBS with constipation (IBS-C), may represent a novel therapeutic for BPS acting through a mechanism involving an inhibition of visceral organ crosssensitization. We showed previously that infusion of dilute protamine sulfate (PS) into the bladder increased sensitivity and permeability in the bladder and colon. PS was infused into the bladder of female rats; sensitivity was assessed via application of von Frey filaments applied to the suprapubic area and the frequency of withdrawal responses was recorded. Colonic sensitivity was measured via visceromotor behavioral response to graded pressures of colorectal distension (CRD).
\end{abstract}

Permeability was measured in vitro via transepithelial electrical resistance (TEER) and conductance $(\mathrm{G})$. Linaclotide $(3 \mu \mathrm{g} / \mathrm{kg}$, p.o.) or vehicle was administered daily for 7 days prior to experiments. Rats treated with PS bladder infusion exhibited visceral hyperalgesia, as shown by a significantly higher response frequency to individual von Frey filaments and increased behavioral responses to CRD. Linaclotide attenuated bladder and colonic hyperalgesia to control levels. PS infusion into the bladder increased bladder and colon permeability measured as a decrease in TEER and increased $G$. Linaclotide significantly inhibited PS-induced colonic hyperpermeability while having no effect on bladder hyperpermeability. Our findings suggest a novel treatment paradigm for GC-C agonism in IBS-C and BPS mediated through a mechanism involving visceral organ crosstalk.

\section{Introduction}

Bladder pain syndrome (BPS) is a chronic pelvic pain condition that affects up to 3-8 million individuals in the United States, the majority of whom are female with symptoms of frequent urination, increased urgency, and pain associated with bladder filling (Held et al., 1990; Berry et al., 2010, 2011; Konkle et al., 2012; Patnaik et al., 2017). As many as $40 \%-60 \%$ of patients diagnosed with BPS also exhibit symptoms of irritable bowel syndrome (IBS), whereas up to $50 \%$ of patients with BPS exhibit symptoms that fulfill the criteria for IBS (Ustinova et al., 2006). IBS is a functional gastrointestinal disorder that is estimated to affect up to $20 \%$ of adolescents and adults in North America and, similar to BPS, exhibits a female predominance (Saito et al., 2002; Longstreth et al., 2006). IBS is characterized by chronic abdominal pain and abnormal bowel habits and is broadly

This work was supported by Ironwood Pharmaceuticals. https://doi.org/10.1124/jpet.118.248567. classified as IBS with diarrhea, IBS with constipation (IBS-C), or mixed IBS (Longstreth, 2005). Patients with IBS commonly suffer from a variety of comorbid regional pain disorders, including BPS. Despite the relatively high comorbidity of both BPS and IBS, their respective etiologies and the mechanisms of symptom overlap in patients diagnosed with both BPS and IBS are not well understood. There is a growing body of evidence suggesting that visceral organ cross-communication is a key contributor to the comorbidity of BPS and IBS. In support of this, studies in rodents have shown that colonic irritation produces irregular micturition patterns and increased urethral sphincter activity in rats (Pezzone et al., 2005). Furthermore, there is evidence that active colonic inflammation induces abnormal bladder detrusor muscle contractility and enhances bladder permeability assessed in vitro in female rats and, conversely, bladder irritation results in increased visceral sensitivity to colonic distension as well as increased colonic permeability in vitro (Bielefeldt et al., 2006; Winnard et al., 2006; Greenwood-Van Meerveld et al., 2015). Although the underlying mechanisms responsible for

ABBREVIATIONS: ANOVA, analysis of variance; BPS, bladder pain syndrome; CRD, colorectal distension; DRG, dorsal root ganglia; G, conductance; GC-C, cuanylate cyclase-C; IBS, irritable bowel syndrome; IBS-C, irritable bowel syndrome with constipation; IR, immunoreactivity; Isc, short circuit current; PBS, phosphate-buffered saline; PD, potential difference; $p E R K$, phosphorylated extracellular signal-regulated kinase; PS, protamine sulfate; TEER, transepithelial electrical resistance. 
visceral organ crosstalk remain incompletely understood, evidence suggests that a number of factors may be involved, including the convergence of sensory neural pathways within the dorsal root ganglia (DRG), spinal cord, and/or brain as well as enhanced epithelial permeability and afferent sensitization (Lavelle et al., 2002; Malykhina et al., 2006; Christianson et al., 2007; Malykhina, 2007; Suckow and Caudle, 2009; Greenwood-Van Meerveld et al., 2015). Although these findings highlight the complex, interconnected pathophysiology of visceral pain disorders, they also point to potential new therapeutic options for the relief of IBS- and BPS-associated visceral pain.

Linaclotide, a synthetic 14-amino-acid agonist peptide of guanylate cyclase-C (GC-C), is approved for treatment of adult patients suffering from IBS-C and chronic idiopathic constipation (Chey et al., 2012; Rao et al., 2012). This peptide resembles the endogenous hormones guanylin and uroguanylin, known to regulate intestinal fluid secretion via GC-C-mediated production of cGMP (Schulz et al., 1990; Camilleri, 2015). Like the endogenous peptides, linaclotide activates GC-C, a transmembrane receptor expressed predominantly on the luminal surface of intestinal epithelial cells, resulting in increased cGMP production (Camilleri, 2015). This increase in intracellular cGMP activates the cGMP/cGMP-dependent protein kinase II/cystic fibrosis transmembrane conductance regulator pathway known to induce fluid secretion and to accelerate transit in animal models (Bryant et al., 2010; Busby et al., 2010). Importantly, linaclotide has been shown to possess antinociceptive properties in models of visceral hypersensitivity (Eutamene et al., 2010; Johnston et al., 2010; Castro et al., 2013). The analgesic effects of linaclotide are independent from its secretory effects but are also linked to cGMP, with GC-C activation stimulating the release of cGMP into the submucosa. This extracellular cGMP is thought to directly act on colonic nociceptors, inhibiting colonic afferent sensitization (Castro et al., 2013). Additional studies have shown that linaclotide inhibits bladder-innervating DRG excitability in a visceral organ crosstalk model of colitis-induced bladder afferent hyperactivity (Han et al., 2011; Grundy et al., 2016). These findings suggest that, in addition to relieving the pain and abnormal bowel habits associated with IBS, GC-C agonism may also improve the urological symptoms in patients with IBS with comorbid BPS. However, it remains to be determined whether linaclotide has any effect on crosstalk induced by a challenge to the urinary bladder rather than the colon. Thus, this study tested the overarching hypothesis that GC-C- mediated mechanisms may be involved in visceral organ crosstalk between the urinary bladder and colon. To address our hypothesis, we investigated the effect of linaclotide in a rodent model of visceral organ cross-sensitization in which administration of dilute protamine sulfate (PS) into the urinary bladder produces bladder and colonic hyperalgesia and causes a significant increase in mucosal permeability in the absence of tissue damage (Greenwood-Van Meerveld et al., 2015).

\section{Materials and Methods}

Animals. A total of 68 female ovariectomized Sprague-Dawley rats $(220-250 \mathrm{~g})$ were purchased from Charles River Laboratories (Wilmington, MA). Rats were single housed within the University of Oklahoma Health Sciences Center Department of Comparative
Medicine' animal facility under controlled temperature $\left(23 \pm 3^{\circ} \mathrm{C}\right)$ and humidity $(30 \%-70 \%)$ with free access to food and water on a standard 12-hour/12-hour light/dark cycle. All animals were acclimated to the facility and laboratory for a minimum of 2 weeks before experimentation. Female rats were used because of ease of urethral catheterization and they were ovariectomized to avoid any effects of hormonal cycling. The experimental protocol was approved by the University of Oklahoma Health Sciences Center Institutional Animal Care and Use Committee (animal protocol 14-151 and 15-028-H).

Experimental Procedure. The experimental procedure is illustrated in Fig. 1. Briefly, animals were randomly assigned to treatment conditions. Rats received an oral administration of either linaclotide ( $3 \mu \mathrm{g} / \mathrm{kg}$, p.o.) or saline vehicle control daily for 7 days. On the sixth day of linaclotide or vehicle dosing, a transurethral catheter was inserted and PS ( $1 \mathrm{mg} / \mathrm{ml}$ in $400 \mu \mathrm{l}$ saline) was infused into the bladder to increase sensitivity not only in the bladder but also in the untreated colon. Sham-treated control animals underwent transurethral catheterization but did not receive PS. After these treatments, bladder or colonic sensitivity was assessed 24 hours after PS infusion (or sham). After colonic distension $(60 \mathrm{~mm} \mathrm{Hg})$, the spinal cord was immediately isolated for quantification of phosphorylated extracellular signalregulated kinase ( $\mathrm{pERK}$ ) expression. In another cohort of PS-treated or sham rats, the bladder and colon were isolated for assessment of permeability. To minimize the number of animals used in this study, blinding was not employed for behavioral assays. This approach ensured that the animals were properly acclimated to the experimenter, which is vital in preventing exposure of animals to novel stressors. However, the experimenter was blinded for the analysis of spinal cord pERK expression.

Administration of PS into the Bladder. Rats were brought to the laboratory and anesthetized with isoflurane (2\%) (Aerrane; Baxter Healthcare, Deerfield, IL) with a steady supply of oxygen for a period of approximately 15 minutes. Body temperature was maintained with a homeothermic blanket, and the PS solution was infused transurethrally as previously described (Lavelle et al., 2002) between 8:00 AM and 10:00 AM. Briefly, the bladder was drained after catheterization using a 20-gauge intravenous catheter (Becton Dickinson Infusion Therapy Systems Inc., Sandy, UT). Animals were monitored at all times during the experimentation and any animals with blood in the urine or solutions were not used. PS at $1 \mathrm{mg} / \mathrm{ml}$ in $400 \mu \mathrm{l}$ saline was slowly infused into the bladder through the catheter. After 10 minutes, the bladder was emptied by applying lower abdominal pressure and then washed with saline $(400 \mu \mathrm{l} \times 3)$, after which the transurethral catheter was removed and animals were returned to their home cages. The bladder and colon were harvested 24 hours after the infusion of PS for the in vitro permeability assessments. Catheterized ovariectomized female rats that were not administered PS served as shamtreated control animals.

Bladder Sensitivity. Bladder sensitivity was assessed via application of von Frey filaments to the animal's suprapubic area (Laird et al., 2001; Rudick et al., 2007; Gosselin et al., 2010; Mohammadi et al., 2016). The frequency of withdrawal responses (response frequency) to individual von Frey filaments with increasing calibrated force $(0.16,0.4$, $1,2,4,8$, and $15 \mathrm{~g}$ ) was recorded. In a separate behavioral room attached to the main laboratory, rats were placed in individual Plexiglas chambers with a stainless-steel wire grid floor and were acclimated to the chambers for a period of 30 minutes, between 10:00 AM and 12:00 PM. Each of the von Frey hairs was applied to the suprapubic region for 1 to 2 seconds for a total of 10 applications/force with a 5 -second rest period between each application. All 10 applications were completed for a single force before changing filaments and the filaments were tested from lowest to highest force. Sharp retraction of the abdomen, immediate licking or grooming, or jumping was considered a positive response to the stimulus.

Colonic Sensitivity. Colonic sensitivity assessment was performed as previously described (Myers and Greenwood-Van Meerveld, 2007) between 10:00 AM and 2:00 PM in a behavioral room attached to the main laboratory. Briefly, rats were brought to the laboratory after 


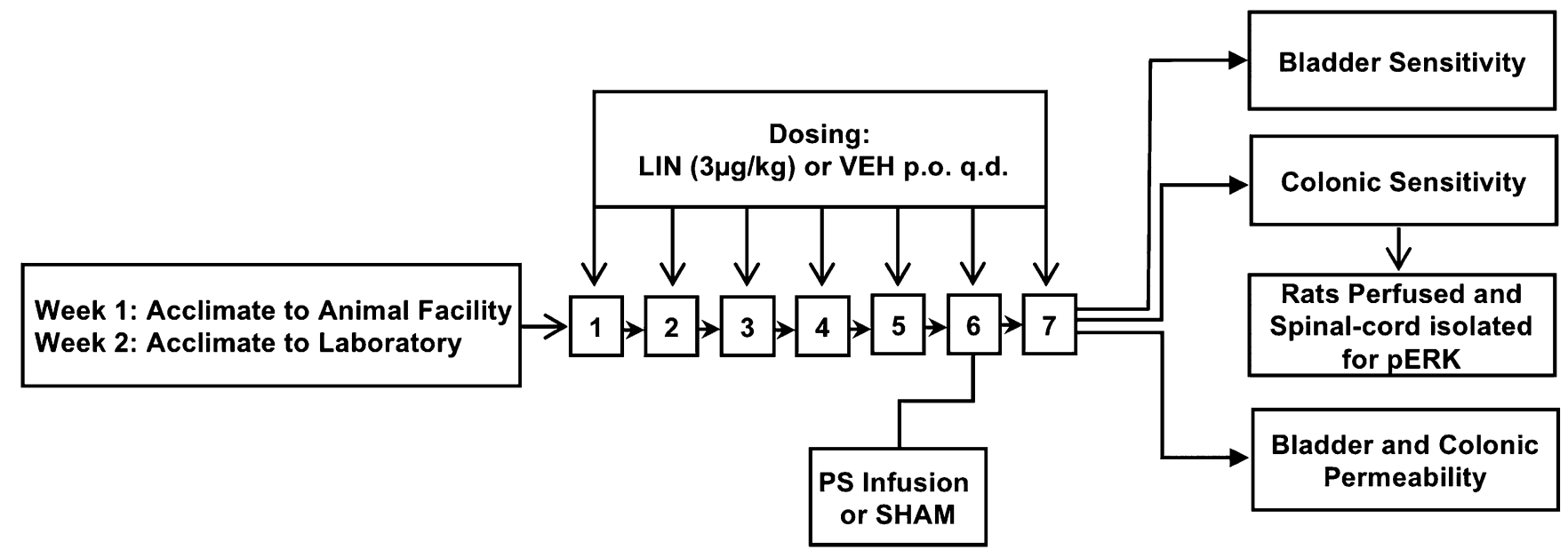

Fig. 1. Rats received an oral administration of linaclotide ( $3 \mu \mathrm{g} / \mathrm{kg}$, p.o.) or saline vehicle control daily for 7 days ( $n=7-9 / \mathrm{group})$. On day 6 , dilute PS $(1 \mathrm{mg} / \mathrm{ml})$ was infused into the urinary bladder via transurethral delivery. Another group of sham-treated control rats $(n=5-9)$ were catheterized but did not receive PS. Bladder and colonic sensitivity was assessed 24 hours after PS in two separate cohorts of animals. Immediately after the final CRD of $60 \mathrm{~mm} \mathrm{Hg}$, the spinal cord was isolated for quantification of pERK expression. In another cohort of PS-treated rats ( $n=7$ to $8 /$ group), the bladder and colon were isolated and permeability was measured in vitro in modified Ussing chambers. LIN, linaclotide; VEH, vehicle.

an overnight fast and anesthetized with $5 \%$ isoflurane. A 5-cm colonic balloon was inserted approximately $11-\mathrm{cm}$ past the anus into the colon, secured to the base of the tail with tape, and attached to a distender series IIR barostat (G \& J Electronics Inc., Toronto, ON, Canada). Colonic sensitivity was assessed via a visceromotor behavioral response to graded pressures (randomized $0-60 \mathrm{~mm} \mathrm{Hg}$ ) of isobaric colorectal distension (CRD) and quantified by the number of abdominal contractions in response to CRD. During this procedure, rats were freely moving and remained in their clean home cage to limit stress. Each constant pressure distension series consisted of a 10-minute basal recording with the balloon inserted but not distended, a 10-minute inflation period, and then a 10-minute rest period between distensions.

Colonic Distension-Induced Mitogen-Activated Protein Kinase pERK Immunoreactivity. Immediately after the final CRD, the rat was anesthetized with isoflurane $(5 \%)$ and underwent transcardial perfusion with ice-cold phosphate-buffered saline (PBS) followed by ice-cold 4\% paraformaldehyde in PBS. After transcardial perfusion, the thoracolumbar (T10-L1) and lumbosacral (L6-S1) regions of the spinal cord were removed and postfixed for 18 hours at $4^{\circ} \mathrm{C}$ in $4 \%$ paraformaldehyde in PBS. After fixation, the spinal cord was cryoprotected in $30 \%$ sucrose in PBS overnight at $4^{\circ} \mathrm{C}$ and then block frozen in optimal cutting temperature compound. Frozen sections $(10 \mu \mathrm{m})$ were cut using a cryostat and placed on Fisherbrand Superfrost Plus microscope slides (Fisher Scientific, Pittsburgh, PA) for future immunohistochemical analysis. Frozen sections were air dried for 20 minutes, postfixed in $4 \%$ paraformaldehyde for 10 minutes, and then washed three times with PBS. The sections were incubated with Biocare's Rodent Block R (cat. no. RBR962H; Biocare Medical, Pacheco, CA) for 20 minutes at room temperature to block nonspecific binding of antibodies. After blocking, the sections were washed three times with $0.2 \%$ Triton X-PBS and then incubated overnight at $4^{\circ} \mathrm{C}$ with anti-phospho-p44/42 mitogen-activated protein kinase (Erk1/2) (Thr202/Tyr204) antibody (pERK; 1:400, no. 4370; Cell Signaling Technology, Danvers, MA) diluted in Biocare Da Vinci Green Diluent (cat. no. PD900H; Biocare Medical). Sections were then washed three times with $0.2 \%$ Triton X-PBS prior to incubation for 1 hour at room temperature with secondary donkey anti-rabbit IgG horseradish peroxidase antibody diluted in Biocare Da Vinci Green Diluent (donkey anti-rabbit IgG; 1:500, cat. no. 711-035-152; Jackson ImmunoResearch Laboratories Inc., West Grove, PA). Sections were washed three times with PBS, incubated with a Betazoid DAB chromogen kit (cat. no. BDB2004H; Biocare Medical) at room temperature for 5 minutes, washed with PBS, and rinsed in deionized water.
Sections were counterstained with hematoxylin. Two sections per region of the spinal cord (T10-T12, T12-L1, L6-S1) per animal were randomly selected and pERK immunoreactive cells in the dorsal horn of each section were identified and counted using a Zeiss Axiovert epifluorescence microscope (Zeiss, Jena, Germany).

Bladder and Colonic Permeability. The rat bladder and colon were isolated postmortem (between 8:00 $\mathrm{AM}$ and 10:00 $\mathrm{AM}$ ) and placed into ice-cold Krebs buffer composed of $120 \mathrm{mM} \mathrm{NaCl}, 6 \mathrm{mM}$ $\mathrm{KCl}, 1.2 \mathrm{mM} \mathrm{MgCl}_{2}, 1.2 \mathrm{mM} \mathrm{H}_{2} \mathrm{PO}_{4}, 2.5 \mathrm{mM} \mathrm{CaCl}_{2}, 14.4 \mathrm{mM} \mathrm{NaHCO}_{3}$, and $11.5 \mathrm{mM}$ glucose, aerated with $95 \% \mathrm{O}_{2}-5 \% \mathrm{CO}_{2}$. The tissue was opened longitudinally and mounted into either biopsy perfusion chambers (bladder) or modified Ussing chambers (colon). Tissues were bathed in oxygenated Kreb's solution at $37^{\circ} \mathrm{C}$ for $30-45$ minutes before experimentation. Permeability was assessed electrophysiologically via measurement of transepithelial electrical resistance (TEER) and conductance (G). To calculate TEER and G, the potential difference (PD) and short circuit current (Isc) were recorded and TEER was calculated using Ohm's law as follows:

$$
I=\frac{P D}{R}
$$

where $R$ represents TEER. Conductance (G) across the tissue being the reciprocal of $R$.

Drug Preparation and Dosing. Linaclotide was supplied by Ironwood Pharmaceuticals (Cambridge, MA) and stored at $-80^{\circ} \mathrm{C}$ until it was prepared for dosing. Linaclotide was prepared at $1 \mu \mathrm{g} / \mathrm{ml}$ in saline vehicle. Rats were weighed and individual dosing volumes were adjusted so that each rat received a human equivalent dose of $3 \mu \mathrm{g} / \mathrm{kg}$ linaclotide or vehicle control (saline, $3.0 \mathrm{ml} / \mathrm{kg}$, p.o.). Oral dosing was performed under isoflurane anesthesia (3-5 minutes), using a 2 -inch stainless-steel feeding tube.

Data Analysis. Bladder and colonic sensitivity were recorded as the number of withdrawal responses and abdominal contractions, respectively. Statistical significance was determined using two-way repeated-measures analysis of variance (ANOVA) followed by a Bonferroni post-test. Spinal cord signaling was recorded as the average number of pERK neurons per each section of the spinal cord. Statistical significance was determined using two-way ANOVA followed by a Bonferroni post-test. Data for TEER and G calculations were generated via Please use EVC499 voltage/current clamp (World Precision Instruments Inc, Sarasota, FL). Transmural PD and Isc were recorded into a laboratory notebook and transferred into an Excel spreadsheet (Microsoft, Redmond, WA). Statistical significance 
was determined using one-way ANOVA with the Tukey post hoc multicomparison test. All test values are expressed as the mean \pm S.E.M. and data analyses were performed using GraphPad Prism (version 6.x; GraphPad Software Inc., La Jolla, CA). For all data, a $95 \%$ confidence interval was used as a measure of statistical significance. $P<0.05$ was considered statistically significant in all tests. A power analysis was performed for each experiment to determine the number of animals needed to reach significance.

\section{Results}

Linaclotide Reverses Bladder and Colonic Hypersensitivity Induced by PS Infusion in the Bladder. In the first series of experiments, we aimed to investigate the hypothesis that linaclotide is capable of disrupting crosstalk between the bladder and colon to inhibit visceral hyperalgesia. To test this hypothesis, we infused PS into the bladder and then assessed visceral sensitivity 24 hours later. As illustrated in Fig. 2A, we found that PS infusion into the bladder significantly increased bladder hypersensitivity, as shown by the increased number of withdrawal responses at 1-15 g compared with sham-treated controls. Linaclotide was found to normalize withdrawal response to low threshold stimulation $(4 \mathrm{~g})$ and significantly decreased bladder hypersensitivity to high-threshold stimuli ( 8 and $15 \mathrm{~g}$ ) in linaclotide-treated rats compared with vehicle-treated rats (Fig. $2 \mathrm{~B})[\mathrm{F}(12,96)=$ 8.49]. Similarly, when dosed for 7 days, linaclotide also significantly decreased colonic hypersensitivity induced by PS infusion into the bladder, evident by the reduced number of abdominal contractions in linaclotide-treated rats compared with vehicle-treated rats, as illustrated in Fig. 2, C and D $[F(6,72)=12.24]$. Of importance to note, linaclotide attenuated visceral hypersensitivity to levels resembling sham controls.

Linaclotide Inhibits Thoracolumbar Spinal Cord Signaling Induced by CRD. A previous report using a model of colonic hypersensitivity showed that linaclotide reduces the signaling of noxious $\mathrm{CRD}$ in the spinal cord, as demonstrated by a reduction in CRD-induced pERK immunoreactivity (IR) in the dorsal horn of the thoracolumbar spinal cord (Castro et al., 2013). Here we tested the hypothesis that linaclotide inhibits CRD-induced spinal cord pERK-IR expression induced by PS infusion into the urinary bladder. We found that linaclotide pretreatment significantly reduced the number of pERK-IR neurons in the thoracolumbar segment of the spinal cord immediately after noxious CRD compared with vehicle-treated rats, as illustrated in Fig. 3, A-D. However, it had no significant effect on the lumbosacral region of the spinal cord (Fig. 3, E and F).

Linaclotide Inhibits Colonic Permeability Induced by PS Infusion in the Bladder. We next considered whether linaclotide reduces visceral hypersensitivity through a mechanism involving inhibition of mucosal hyperpermeability. Consistent with our previous reports (Greenwood-Van Meerveld et al., 2015), PS infusion into the bladder induced a

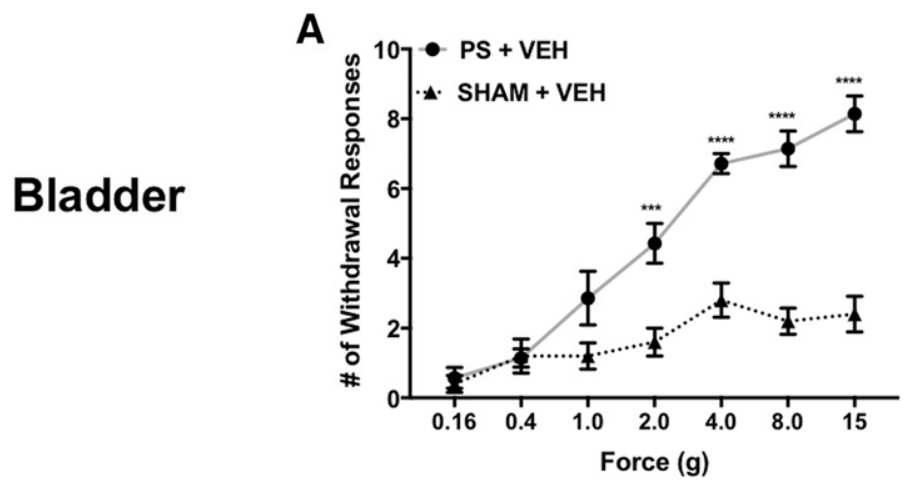

C

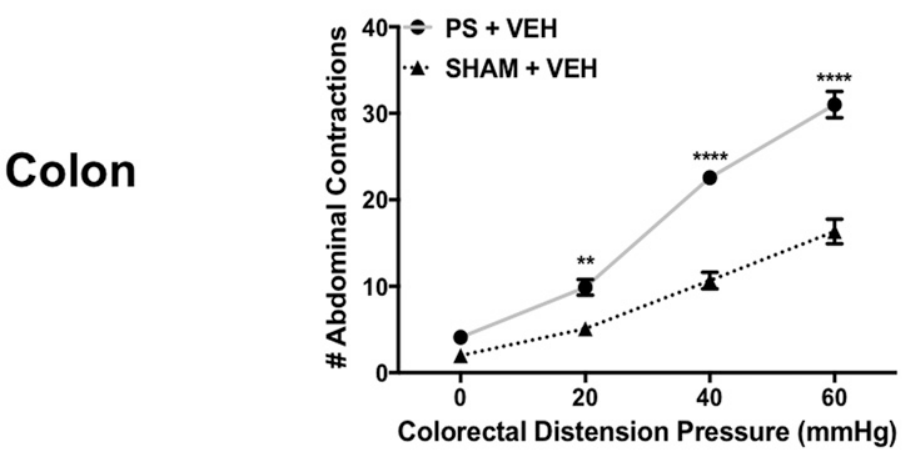

B

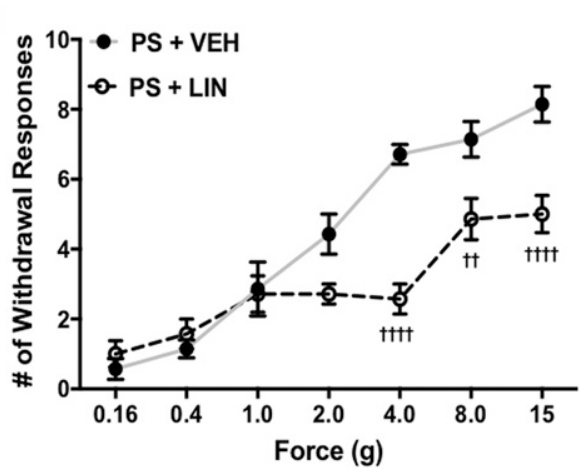

D

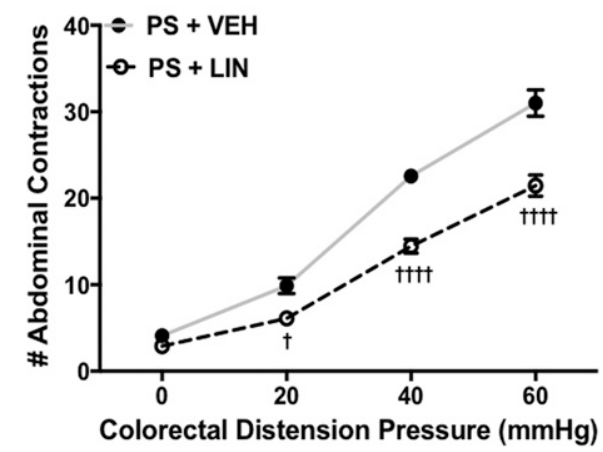

Fig. 2. (A) PS was infused into the bladder and 24 hours later, we observed a significant increase in the number of withdrawal responses to suprapubic stimulation in vehicle-treated rats $(n=7)$ compared with sham controls $(n=5)$. (B) In rats $(n=7)$, pretreatment with linaclotide for 7 days significantly decreased the average withdrawal response frequencies to suprapubic stimulation compared with vehicle-treated animals. (C) An increase in colonic sensitivity was shown as an exaggerated visceromotor response to CRD in vehicle-treated rats $(n=9)$ compared with sham controls $(n=9)$. (D) Pretreatment with linaclotide for 7 days $(n=9)$ reversed the effect of PS-induced colonic hypersensitivity, as shown in the significant reduction of the visceromotor response to CRD. Data are expressed as the mean \pm S.E.M. Statistical significance was determined using two-way repeated-measures ANOVA followed by a Bonferroni post-test. ${ }^{* *} P<0.01 ; * * * P<0.001 ; * * * * P<0.0001$ (compared with sham controls plus vehicle); ${ }^{\dagger} P<0.05 ;{ }^{\dagger} P P<0.01$; ${ }^{\dagger+\dagger \dagger} P<0.0001$ (compared with PS plus vehicle). LIN, linaclotide; VEH, vehicle. 

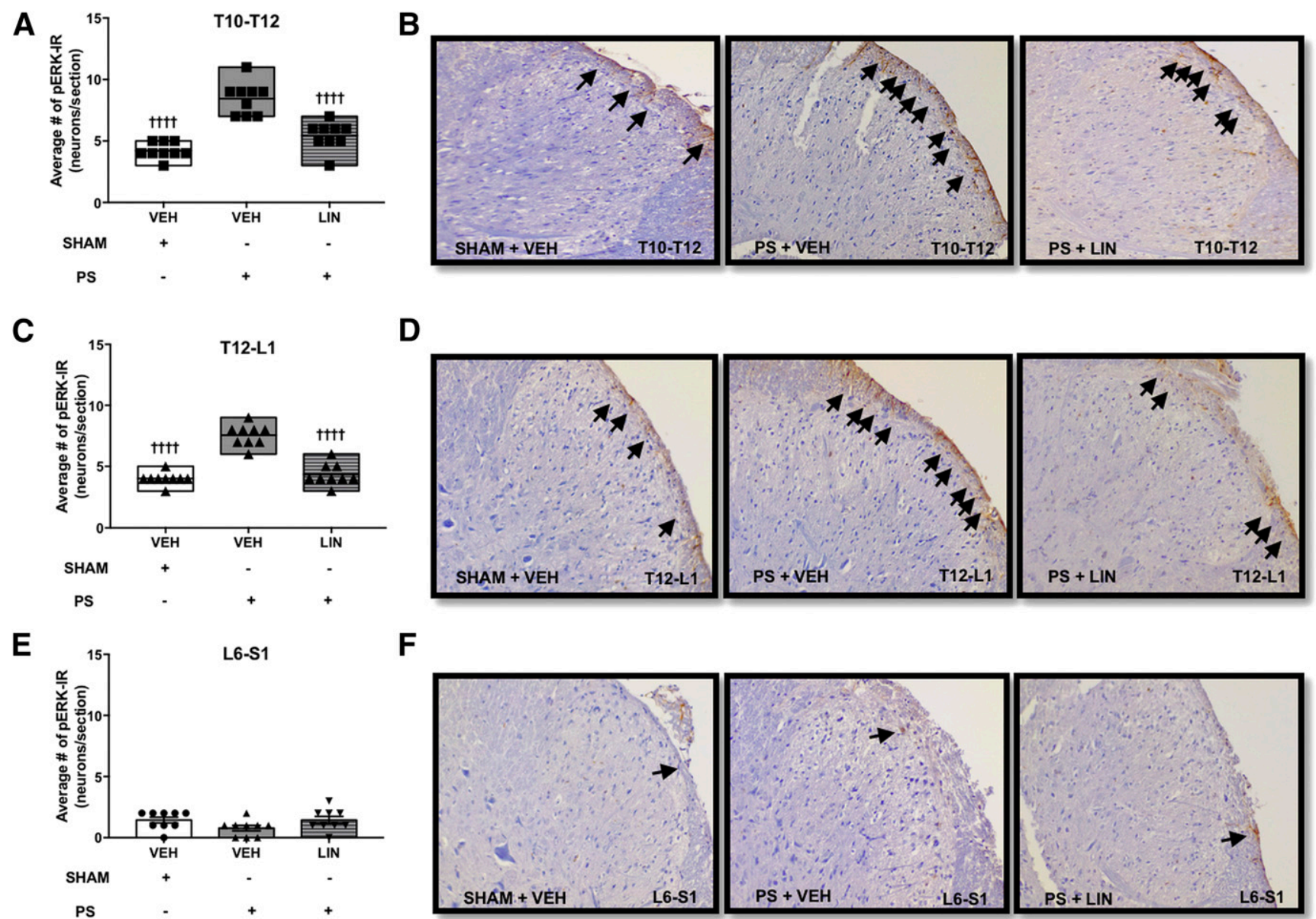

Fig. 3. PS was infused into the bladder and 24 hours after the infusion, there was a significant increase in nociceptive signaling in the dorsal horn of the spinal cord in response to noxious CRD. (A-F) Administration of linaclotide significantly reduced the number of pERK-IR-activated neurons (black arrows) in the T10-T12 (A and B) and T12-L1 (C and D) spinal cord compared with vehicle-treated rats, but it had no significant effect on L6-S1 of the spinal cord ( $\mathrm{E}$ and $\mathrm{F}) .(n=\mathrm{X} / \mathrm{X}$ denotes the number of animals/number of sections). Data are expressed as the mean \pm S.E.M. Statistical significance was determined using two-way ANOVA followed by a Bonferroni post-test. ${ }^{\dagger \dagger} P<0.0001$ (compared with PS plus vehicle). LIN, linaclotide; VEH, vehicle.

marked increase in bladder permeability, as shown by a significant decrease in TEER and a significant increase in $\mathrm{G}$ compared with sham animals $[\mathrm{F}(2,9)=54.93]$, as illustrated in Fig. 4. To probe the effects of linaclotide on PS-induced bladder and colonic hyperpermeability, we isolated the bladder and colon from rats pretreated with either linaclotide or vehicle for 6 days prior to PS infusion into the bladder. Findings from these animals were compared with shamtreated rats. We found that compared with vehicle-treated animals, linaclotide had a significant inhibitory effect on colonic hyperpermeability induced by infusion of PS into the bladder. There was a significant decrease in colonic TEER $[\mathrm{F}(2,39)=25.83]$ and a significant increase in colonic $\mathrm{G}$ $[\mathrm{F}(2,39)=22.1]$ in colonic tissue isolated from rats 24 hours after PS infusion in the bladder compared with sham-treated vehicle controls. Linaclotide was found to reverse this increase in colonic permeability, as determined by a significant increase in TEER (Fig. 4A) $[\mathrm{F}(2,39)=0.506]$ and a significant decrease in $\mathrm{G}$ (Fig. 4B) [F $(2,39)=8.002]$. In contrast with our findings in the colon, the magnitude of the decrease in TEER (Fig. 4C) and increase in G (Fig. 4D) in the bladder in rats pretreated with linaclotide was not statistically different from vehicletreated controls, suggesting that there was no reversal of PS-induced permeability in the bladder by linaclotide.

\section{Discussion}

This study provides experimental evidence that GC-C-mediated mechanisms play a pivotal role in visceral organ crosstalk. We demonstrate that PS infusion into the bladder induced hypersensitivity of the bladder and of the undamaged colon. These pathophysiological responses after PS infusion into the bladder were inhibited by pretreatment with linaclotide, a selective and potent GC-C agonist. Specifically, linaclotide, at a clinically relevant/meaningful dose, attenuated bladder and colonic hyperalgesia to levels resembling controls. To uncover the potential mechanism(s) underlying the antinociceptive effect of linaclotide, we investigated the hypothesis that an attenuation of visceral hypersensitivity by linaclotide may result from normalization in mucosal hyperpermeability, which in turn leads to a reduction in the processing of noxious CRD in the thoracolumbar spinal cord. In this study, we found that PS administered into the bladder significantly increased bladder and colon permeability. Furthermore, in support of our hypothesis, we demonstrated that linaclotide significantly inhibited PS-induced colonic permeability while having no effect on bladder hyperpermeability. In animals treated with linaclotide, we also observed a reduction in colonic distensioninduced pERK immunoreactivity in the spinal cord in animals 
A

Colon

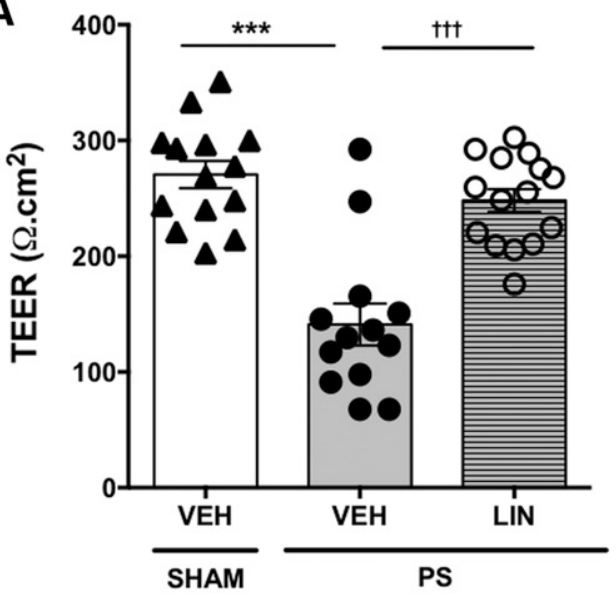

C

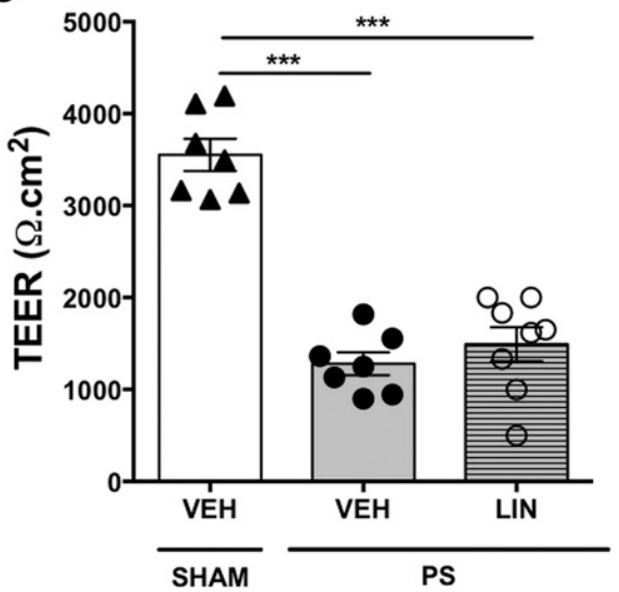

B

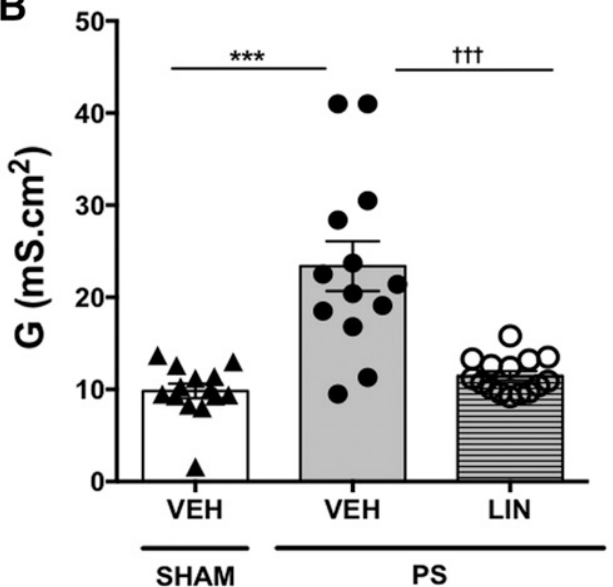

D

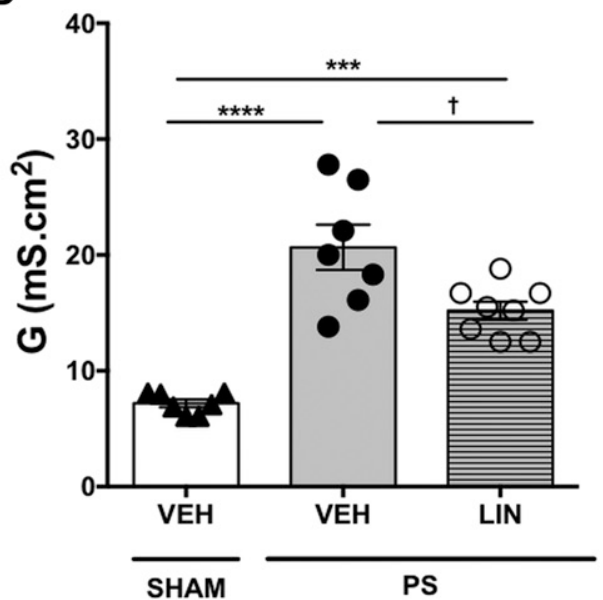

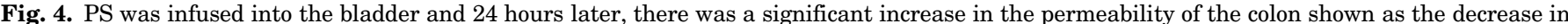

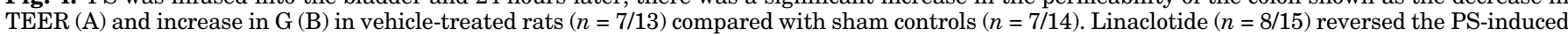

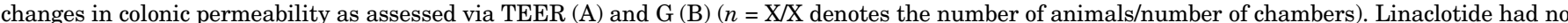

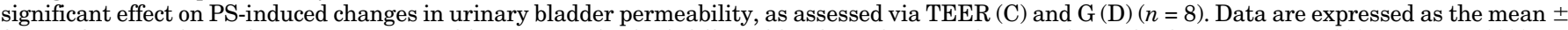

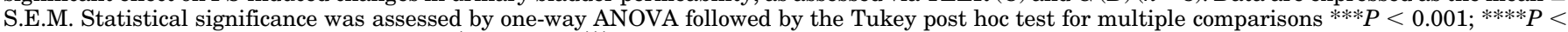
0.0001 (compared with sham plus vehicle); ${ }^{\dagger} P<0.005 ;{ }^{\dagger \dagger} P<0.001$ (compared with PS plus vehicle). LIN, linaclotide; VEH, vehicle.

receiving PS infusion into the bladder. These novel findings, suggest that linaclotide is capable of attenuating visceral pain emanating from the bladder and colon through mechanisms that appear to involve, at least in part, effects on visceral organ permeability and spinal cord neuronal processing. Importantly, these findings offer further insight into novel treatment options for patients with comorbid IBS-C and BPS.

Although there is well-known comorbidity between IBS and BPS, the etiology of these disorders is unknown and treatment options for BPS patients diagnosed with debilitating and painful symptoms remain unsatisfactory. Previously, linaclotide was shown to elicit antinociceptive effects in rodent models of colonic hypersensitivity induced by stress or after acute colitis via a mechanism involving inhibition of stretch-sensitive high-threshold mechanosensitive afferents at the level of the gut (Eutamene et al., 2010; Castro et al., 2013; Feng et al., 2013). Here we set out to investigate whether GC-C agonism is capable of inhibiting colonic hypersensitivity induced by a challenge to another visceral organ (namely, the bladder). In this study, we employed a rodent model in which bladder infusion of dilute PS increased colonic sensitivity in the absence of any alterations to the histologic appearance of the colon and only minimal damage to the bladder urothelium (Greenwood-Van Meerveld et al., 2015). Our results demonstrate that linaclotide inhibits PS-induced colonic hypersensitivity. Based on this novel observation, we hypothesize that linaclotide-mediated attenuation of visceral hyperalgesia appears to extend to noninflammatory conditions.

In this study, we aimed to probe the mechanism(s) by which linaclotide attenuates visceral hypersensitivity induced by visceral organ cross-sensitization. A growing body of evidence suggests that overlapping symptomology of BPS and IBS is possibly linked to visceral organ crosstalk in which increases in epithelial permeability drive abnormalities in the sensitivity of distant visceral organs. Abnormal barrier function and secretory activity have been implicated in patients with IBS (Camilleri et al., 2012; Bischoff et al., 2014). Evidence in the literature points to the concept that increased colonic permeability may lead to sensitization of sensory afferents, increased spinal neuronal excitability, and even central sensitization (Zhou and Verne, 2011; Camilleri et al., 2012; 
Hurst et al., 2015). However, other findings supporting a role of abnormal bladder permeability producing BPS symptoms are controversial and questions remain as to whether an increase in urothelial permeability is causal or an effect of the increased pain. Previously we found that there was an increase permeability of not only the bladder but also the undamaged colon after acute very mild disruption of the bladder urothelium using dilute PS (Greenwood-Van Meerveld et al., 2015). Here urinary bladder or colonic permeability was rapidly assessed electrophysiologically in modified Ussing chamber preparations via measurements of transepithelial PD and Isc to calculate TEER via Ohm's law. Furthermore, PS infusion into the bladder stimulated bladder and colonic hyperalgesia (Baker et al., 2017). Taken together, our previous work supports the concept that linaclotide treatment may have reduced bladder and colon hypersensitivity by a reversal of PS-induced barrier dysfunction. In this study, we found that linaclotide inhibited colonic mucosal hyperpermeability induced by PS infusion into the bladder. When combined with the antinociceptive effect of linaclotide in the colon, our data provide supporting proof that increased permeability may have played a role in colonic hyperalgesia. However, our data revealed an additional element in the control of bladder hypersensitivity. We found that despite an inhibition of referred bladder hyperalgesia, linaclotide showed no inhibitory effect on the increase in bladder TEER and G. This disconnect between the inhibitory effect of linaclotide on bladder hypersensitivity and lack of a reduction in urothelial permeability implies that an additional mechanism is responsible for antinociceptive effects of GC-C agonism in the bladder. There is evidence for systemic effects produced by the loss of barrier function in the bladder affecting the remote organ via the release of inflammatory cytokines from mast cells (Grover et al., 2011). Since linaclotide elicits its pharmacological effects locally in the colon (Bryant et al., 2010; Busby et al., 2010), potential systemic effects induced by bladder hyperpermeability may be insensitive to GC-C agonism. A large body of evidence now supports an underlying mechanism of visceral organ crosstalk based on convergence of sensory neural pathways within the DRG, spinal cord, and/or brain, leading to peripheral and central sensitization to produce visceral hypersensitivity (Lavelle et al., 2002; Malykhina et al., 2006; Christianson et al., 2007; Malykhina, 2007; Suckow and Caudle, 2009; Greenwood-Van Meerveld et al., 2015). In this study, we showed that linaclotide, despite being locally restricted to the colon, inhibits bladder-colon visceral organ cross-sensitization activated by PS infusion into the bladder. Thus, the effects of linaclotide appear mediated by dual mechanisms involving not only effects on intestinal barrier function but importantly, inhibition of peripheral sensory signaling to the spinal cord to reduce central sensitization. Here we showed that in rats with PS infused in the bladder, linaclotide decreased CRD-induced $\mathrm{pERK}$ expression in the thoracolumbar spinal cord, specifically in the superficial lamina of the dorsal horn of the rat, which is the major site of afferent terminations responding to nociceptive input from the colon and bladder. Our data build upon previous studies showing a decrease in CRD-induced pERK expression by linaclotide in a rodent model of postinflammatory colonic hyperalgesia (Castro et al., 2013). Taken together, these results show that the attenuation of visceral hypersensitivity in linaclotide-treated rats occurs in part via a reduction in the number of activated neurons in the dorsal horn of the thoracolumbar spinal cord.

In conclusion, our findings demonstrate that GC-C agonism plays a novel role in visceral organ cross-communication between the bladder and colon, a mechanism not previously linked to linaclotide. These preclinical findings illustrate that linaclotide has effects that extend beyond the colon and the data provide a rationale for clinical studies to further investigate the therapeutic benefit of linaclotide in patients with IBS-C and comorbid BPS.

\section{Acknowledgments}

We thank Anthony Johnson for thoughtful comments and suggestions on the final version of the manuscript.

\section{Authorship Contributions}

Participated in research design: Mohammadi, Ligon, Ge, Kurtz, Higgins, Hannig, Greenwood-Van Meerveld, Silos-Santiago.

Conducted experiments: Mohammadi, Ligon.

Performed data analysis: Mohammadi, Ligon.

Wrote or contributed to the writing of the manuscript: Mohammadi, Ligon, Ge, Kurtz, Higgins, Hannig, Greenwood-Van Meerveld.

\section{References}

Baker Q, Mohammadi E, Ligon C, and Greenwood-Van Meerveld B (2017) Pd01-12 bladder hyperpermeability induces persistent visceral pain: a novel mechanism for visceral organ crosstalk. J Urol 197:e50.

Berry SH, Bogart LM, Pham C, Liu K, Nyberg L, Stoto M, Suttorp M, and Clemens JQ (2010) Development, validation and testing of an epidemiological case definition of interstitial cystitis/painful bladder syndrome. J Urol 183:1848-1852

Berry SH, Elliott MN, Suttorp M, Bogart LM, Stoto MA, Eggers P, Nyberg L, and Clemens JQ (2011) Prevalence of symptoms of bladder pain syndrome/interstitial cystitis among adult females in the United States. J Urol 186:540-544.

Bielefeldt K, Lamb K, and Gebhart GF (2006) Convergence of sensory pathways in the development of somatic and visceral hypersensitivity. Am J Physiol Gastrointest Liver Physiol 291:G658-G665.

Bischoff SC, Barbara G, Buurman W, Ockhuizen T, Schulzke JD, Serino M, Tilg H, Watson A, and Wells JM (2014) Intestinal permeability-a new target for disease prevention and therapy. BMC Gastroenterol 14:189.

Bryant AP, Busby RW, Bartolini WP, Cordero EA, Hannig G, Kessler MM, Pierce CM, Solinga RM, Tobin JV, Mahajan-Miklos S, et al. (2010) Linaclotide is a potent and selective guanylate cyclase $\mathrm{C}$ agonist that elicits pharmacological ef fects locally in the gastrointestinal tract. Life Sci 86:760-765.

Busby RW, Bryant AP, Bartolini WP, Cordero EA, Hannig G, Kessler MM, Mahajan-Miklos S, Pierce CM, Solinga RM, Sun LJ, et al. (2010) Linaclotide, through activation of guanylate cyclase $\mathrm{C}$, acts locally in the gastrointestinal tract to elicit enhanced intestinal secretion and transit. Eur J Pharmacol 649: $328-335$

Camilleri M (2015) Guanylate cyclase C agonists: emerging gastrointestinal therapies and actions. Gastroenterology 148:483-487.

Camilleri M, Madsen K, Spiller R, Greenwood-Van Meerveld B, and Verne GN (2012) Intestinal barrier function in health and gastrointestinal disease [published correction appears in Neurogastroenterol Motil (2012) 24:976]. Neurogastroenterol Motil 24:503-512.

Castro J, Harrington AM, Hughes PA, Martin CM, Ge P, Shea CM, Jin H, Jacobson S, Hannig G, Mann E, et al. (2013) Linaclotide inhibits colonic nociceptors and relieves abdominal pain via guanylate cyclase-C and extracellular cyclic guanosine 3',5'-monophosphate. Gastroenterology 145:1334-1346.

Chey WD, Lembo AJ, Lavins BJ, Shiff SJ, Kurtz CB, Currie MG, MacDougall JE, Jia XD, Shao JZ, Fitch DA, et al. (2012) Linaclotide for irritable bowel syndrome with constipation: a 26-week, randomized, double-blind, placebo-controlled trial to evaluate efficacy and safety. Am J Gastroenterol 107:1702-1712.

Christianson JA, Liang R, Ustinova EE, Davis BM, Fraser MO, and Pezzone MA (2007) Convergence of bladder and colon sensory innervation occurs at the primary afferent level. Pain 128:235-243.

Eutamene H, Bradesi S, Larauche M, Theodorou V, Beaufrand C, Ohning G, Fioramonti J, Cohen M, Bryant AP, Kurtz C, et al. (2010) Guanylate cyclase $\mathrm{C}$-mediated antinociceptive effects of linaclotide in rodent models of visceral pain. Neurogastroenterol Motil 22:312-e84.

Feng B, Kiyatkin ME, La JH, Ge P, Solinga R, Silos-Santiago I, and Gebhart GF (2013) Activation of guanylate cyclase-C attenuates stretch responses and sensitization of mouse colorectal afferents. J Neurosci 33:9831-9839.

Gosselin RD, O'Connor RM, Tramullas M, Julio-Pieper M, Dinan TG, and Cryan JF (2010) Riluzole normalizes early-life stress-induced visceral hypersensitivity in rats: role of spinal glutamate reuptake mechanisms. Gastroenterology 138: $2418-2425$

Greenwood-Van Meerveld B, Mohammadi E, Tyler K, Van Gordon S, Parker A Towner R, and Hurst R (2015) Mechanisms of visceral organ crosstalk: importance of alterations in permeability in rodent models. J Urol 194:804-811. 
Grover S, Srivastava A, Lee R, Tewari AK, and Te AE (2011) Role of inflammation in bladder function and interstitial cystitis. Ther Adv Urol 3:19-33.

Grundy LA, Garcia-Carraballo S, Maddern J, Rychkov G, Hannig G, Kurtz CB, SilosSantiago A, and Brierley S (2016) 561 chronic oral administration of the guanylate cyclase-C agonist linaclotide attenuates colitis induced bladder afferent hyperactivity. Gastroenterology 150:S118-S119.

Han X, Mann E, Gilbert S, Guan Y, Steinbrecher KA, Montrose MH, and Cohen MB (2011) Loss of guanylyl cyclase C (GCC) signaling leads to dysfunctional intestinal barrier. PLoS One 6: 16139.

Held PJ, Hanno PM, Wein AJ, Pauly MV, and Cahn MA (1990) Epidemiology of interstitial cystitis: 2, in Interstitial Cystitis (Hanno PM, Staskin DR, Krane RJ, and Wein AJ eds) pp 29-48, Springer, London.

Hurst RE, Greenwood-Van Meerveld B, Wisniewski AB, VanGordon S, Lin H, Kropp $\mathrm{BP}$, and Towner RA (2015) Increased bladder permeability in interstitial cystitis/painful bladder syndrome. Transl Androl Urol 4:563-571.

Johnston JM, Kurtz CB, Macdougall JE, Lavins BJ, Currie MG, Fitch DA, O'Dea C, Baird M, and Lembo AJ (2010) Linaclotide improves abdominal pain and bowel habits in a phase IIb study of patients with irritable bowel syndrome with constipation. Gastroenterology 139:1877-1886.

Konkle KS, Berry SH, Elliott MN, Hilton L, Suttorp MJ, Clauw DJ, and Clemens JQ (2012) Comparison of an interstitial cystitis/bladder pain syndrome clinical cohort with symptomatic community women from the RAND Interstitial Cystitis Epidemiology study. J Urol 187:508-512.

Laird JM, Martinez-Caro L, Garcia-Nicas E, and Cervero F (2001) A new model of visceral pain and referred hyperalgesia in the mouse. Pain 92:335-342.

Lavelle J, Meyers S, Ramage R, Bastacky S, Doty D, Apodaca G, and Zeidel ML (2002) Bladder permeability barrier: recovery from selective injury of surface epithelial cells. Am J Physiol Renal Physiol 283:F242-F253.

Longstreth GF (2005) Definition and classification of irritable bowel syndrome: current consensus and controversies. Gastroenterol Clin North Am 34:173-187.

Longstreth GF, Thompson WG, Chey WD, Houghton LA, Mearin F, and Spiller RC (2006) Functional bowel disorders. Gastroenterology 130:1480-1491.

Malykhina AP (2007) Neural mechanisms of pelvic organ cross-sensitization. Neuroscience 149:660-672.

Malykhina AP, Qin C, Greenwood-van Meerveld B, Foreman RD, Lupu F, and Akbarali HI (2006) Hyperexcitability of convergent colon and bladder dorsal root ganglion neurons after colonic inflammation: mechanism for pelvic organ cross-talk. Neurogastroenterol Motil 18:936-948.

Mohammadi E, Prusator DK, Healing E, Hurst R, Towner RA, Wisniewski AB and Greenwood-Van Meerveld B (2016) Sexually dimorphic effects of early life stress in rat pups on urinary bladder detrusor muscle contractility in adulthood. Biol Sex Differ 7:8.
Myers B and Greenwood-Van Meerveld B (2007) Corticosteroid receptor-mediated mechanisms in the amygdala regulate anxiety and colonic sensitivity. Am J Physiol Gastrointest Liver Physiol 292:G1622-G1629.

Patnaik SS, Laganà AS, Vitale SG, Butticè S, Noventa M, Gizzo S, Valenti G, Rapisarda AMC, La Rosa VL, Magno C, et al. (2017) Etiology, pathophysiology and biomarkers of interstitial cystitis/painful bladder syndrome. Arch Gynecol Obstet 295:1341-1359.

Pezzone MA, Liang R, and Fraser MO (2005) A model of neural cross-talk and irritation in the pelvis: implications for the overlap of chronic pelvic pain disorders. Gastroenterology 128:1953-1964.

Rao S, Lembo AJ, Shiff SJ, Lavins BJ, Currie MG, Jia XD, Shi K, MacDougall JE, Shao JZ, Eng P, et al. (2012) A 12-week, randomized, controlled trial with a 4-week randomized withdrawal period to evaluate the efficacy and safety of linaclotide in irritable bowel syndrome with constipation. Am J Gastroenterol 107:1714-1724; quiz p 1725.

Rudick CN, Chen MC, Mongiu AK, and Klumpp DJ (2007) Organ cross talk modulates pelvic pain. Am J Physiol Regul Integr Comp Physiol 293:R1191-R1198.

Saito YA, Schoenfeld P, and Locke GR, 3rd (2002) The epidemiology of irritable bowel syndrome in North America: a systematic review. Am J Gastroenterol 97: 1910-1915.

Schulz S, Green CK, Yuen PS, and Garbers DL (1990) Guanylyl cyclase is a heatstable enterotoxin receptor. Cell 63:941-948.

Suckow SK and Caudle RM (2009) NMDA receptor subunit expression and PAR2 receptor activation in colospinal afferent neurons (CANs) during inflammation induced visceral hypersensitivity. Mol Pain 5:54

Ustinova EE, Fraser MO, and Pezzone MA (2006) Colonic irritation in the rat sensitizes urinary bladder afferents to mechanical and chemical stimuli: an afferent origin of pelvic organ cross-sensitization. Am J Physiol Renal Physiol 290: F1478-F1487.

Winnard KP, Dmitrieva N, and Berkley KJ (2006) Cross-organ interactions between reproductive, gastrointestinal, and urinary tracts: modulation by estrous stage and involvement of the hypogastric nerve. Am J Physiol Regul Integr Comp Physiol 291:R1592-R1601.

Zhou Q and Verne GN (2011) New insights into visceral hypersensitivity-clinical implications in IBS. Nat Rev Gastroenterol Hepatol 8:349-355.

Address correspondence to: Dr. Beverley Greenwood-Van Meerveld, Oklahoma Center for Neuroscience, University of Oklahoma Health Science Center, BRC 272, 975 NE 10th St., Oklahoma City, OK 73104. E-mail: beverley-greenwood@ouhsc.edu 